\title{
An Exact Solution of the Convective Couette Flow Under the Parabolic Heating Condition at the Lower Boundary of a Fluid Layer
}

\author{
V. V. Privalova, ${ }^{1,2)}$ and E. Yu. Prosviryakov ${ }^{1, \text { b) }}$ \\ ${ }^{1}$ Institute of Engineering Science, Ural Branch of the Russian Academy of Sciences, \\ 34 Komsomolskaya St., Ekaterinburg, 620049, Russia \\ ${ }^{2}$ B. N. Yeltsin Ural Federal University, 19 Mira St., Ekaterinburg, 620002, Russia

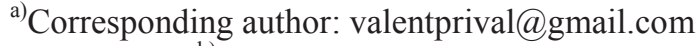 \\ b) evgen_pros@mail.ru
}

\begin{abstract}
A new exact solution of the Navier-Stokes equation for the convective flow of an infinite horizontal layer of a viscous incompressible fluid is obtained. Parabolic heating is specified at one of the layer boundaries. The other boundary of the fluid layer is permeable. The obtained exact solution generalizes the isothermal Couette flow and the Ostroumov- Birikh convective analogues. The proposed class of exact solutions takes into account the horizontal change in hydrodynamic fields. The obtained solution analysis for the velocity and temperature fields is presented. The possibility of the existence of stagnation points and counterflow areas in a moving layer of a non-isothermal viscous incompressible fluid is demonstrated.
\end{abstract}

\section{PROBLEM STATEMENT}

We consider equations of plane motion of a viscous incompressible fluid. These equations describe the effect of temperature on the distribution of hydrodynamic fields in the Boussinesq approximation $[1,2]$ as

$$
\begin{gathered}
\frac{\partial V_{x}}{\partial t}+V_{x} \frac{\partial V_{x}}{\partial x}+V_{z} \frac{\partial V_{x}}{\partial z}=-\frac{\partial P}{\partial x}+v\left(\frac{\partial^{2} V_{x}}{\partial x^{2}}+\frac{\partial^{2} V_{x}}{\partial z^{2}}\right), \\
\frac{\partial V_{z}}{\partial t}+V_{x} \frac{\partial V_{z}}{\partial x}+V_{z} \frac{\partial V_{z}}{\partial z}=-\frac{\partial P}{\partial z}+v\left(\frac{\partial^{2} V_{z}}{\partial x^{2}}+\frac{\partial^{2} V_{z}}{\partial z^{2}}\right)+g \beta T, \\
\frac{\partial T}{\partial t}+V_{x} \frac{\partial T}{\partial x}+V_{z} \frac{\partial T}{\partial z}=\chi\left(\frac{\partial^{2} T}{\partial x^{2}}+\frac{\partial^{2} T}{\partial z^{2}}\right) \\
\frac{\partial V_{x}}{\partial x}+\frac{\partial V_{z}}{\partial z}=0 .
\end{gathered}
$$

Here, $V_{x}$ and $V_{z}$ are the velocities parallel to the corresponding coordinate axes of a rectangular Cartesian coordinate system; $P$ is the deviation of pressure from hydrostatic, taken relative to the constant average fluid density $\rho ; T$ is the deviation from the average temperature; $v, \chi$ are the dissipative coefficients of kinematic viscosity and thermal diffusivity of a fluid, respectively; $g$ is gravity acceleration; $\beta$ is the temperature coefficient of volume expansion [2]. 
Equations (1) describe the motion of a horizontal fluid layer parallel to the $O x$ axis. The lower boundary of the fluid layer is assumed to be absolutely solid, and the upper boundary is permeable, i.e. nonzero vertical velocity $V_{z}$ in the layer is taken into account. We assume that the fluid flow is steady and that all the velocity, temperature, and pressure fields are time-independent.

A solution to the Oberbeck-Boussinesq system (1) will be sought in the following form [3-6]:

$$
\begin{gathered}
V_{x}=U(z)+x u(z), \quad V_{z}=w(z), \\
P=P_{0}(z)+\frac{x^{2}}{2} P_{1}(z), \quad T(z)=T_{0}(z)+\frac{x^{2}}{2} T_{1}(z) .
\end{gathered}
$$

Solution (2) generalizes the class of exact solutions for velocities linearly increasing along the horizontal coordinates, which started to be systematically studied in [7] by Lin for problems of magnetohydrodynamics problems, although Euler had already used the representation (2) for velocities describing gas motion [8]. The review [9] provides a detailed analysis of exact isothermal solutions, shows that the pressure components are related to the coefficients found in (2) for velocities. Thus, there is a relationship between pressure and velocity. Similarly, a relationship between velocities and temperature is established.

We substitute expressions (2) into the system of equations (1) and arrive at an equation system to determine the unknown functions involved in the class of exact solutions represented in (2). We write the obtained system in the order of further integration as

$$
\begin{gathered}
\frac{d^{2} T_{1}}{d z^{2}}=0, \frac{d^{2} T_{0}}{d z^{2}}+T_{1}=0, \\
\frac{d P_{1}}{d z}=g \beta T_{1}, v \frac{d^{2} u}{d z^{2}}=P_{1}, v \frac{d^{2} U}{d z^{2}}=0, \\
\frac{d w}{d z}+u=0, \frac{d P_{0}}{d z}=g \beta T_{0}+v \frac{d^{2} w}{d z^{2}} .
\end{gathered}
$$

The general solution of system (3) is polynomial functions of the variable $z$ up to the fifth order, inclusive.

\section{A BOUNDARY VALUE PROBLEM WITH THE PARABOLIC HEATING OF THE LOWER BOUNDARY}

We formulate boundary conditions to calculate the integration constants. These boundary conditions determine the convective Couette flow in an infinite layer. The no-slip conditions are satisfied at the lower (solid) boundary,

$$
V_{x}(0)=V_{z}(0)=0 \text {. }
$$

The upper boundary is defined by the equation of the plane $z=h$. An inhomogeneous velocity distribution is set at this boundary as

$$
V_{x}(h)=W+\Omega x
$$

For the description of convective motion, we will assume that the upper boundary is isothermal, with the reference temperature

$$
T(h)=0 .
$$

Heating at the lower boundary is set according to the following law: 


$$
T(0)=A+\frac{x^{2}}{2} \frac{B}{l^{2}}
$$

Here, $l$ is a characteristic horizontal scale, which should be equal to infinity since we consider an infinite fluid layer along the $x$ coordinate. However, in nature, physical processes have finite dimensions. The introduction of a finite number $l$, which plays the role of a characteristic horizontal scale, provides an estimate of the horizontal dimension of the fluid layer. For this horizontal dimension, the boundary conditions at the lateral boundaries can be neglected. Therefore, for such values of $l$ in solving boundary value problems, an infinite fluid layer can be used as the abstract mathematical object.

Taking into account the assumed form of solution (2) and the linearity of the conditions at the boundaries, we obtain the following conditions for $z=0$ :

$$
\begin{gathered}
U(0)=u(0)=w(0)=0, \\
T_{0}(0)=A, T_{1}(0)=\frac{B}{l^{2}} .
\end{gathered}
$$

The following equalities hold at the upper boundary:

$$
\begin{aligned}
& U(h)=W, u(h)=\Omega, \\
& T_{0}(h)=0, T_{1}(h)=0 .
\end{aligned}
$$

At the lower boundary, the boundary conditions for reduced pressure are written as

$$
P_{0}(h)=S, P_{1}(h)=0 \text {. }
$$

System (3) with the given boundary conditions (4)-(8) is solved as follows:

$$
\begin{gathered}
U=W Z, u=Z\left[\Omega-\frac{B g \delta^{2} h \beta}{24 v}\left(-3+6 Z-4 Z^{2}+Z^{3}\right)\right], \\
w=Z^{2}\left[\frac{B g \beta h^{2} \delta^{2}}{240 v}\left(-15+20 Z-10 Z^{2}+2 Z^{3}\right)-\frac{1}{2} h \Omega\right], \\
T_{0}=\frac{1}{6}(Z-1)\left[B(Z-2) Z \delta^{2}-6 A\right], T_{1}=-\frac{B \delta^{2}}{h^{2}}(Z-1), \\
P_{0}=S+\frac{1}{24}(Z-1)\left[-12 A g \beta h(Z-1)+B g \beta \delta^{2} h\left(1+4 Z-6 Z^{2}+2 Z^{3}\right)-24 v \Omega\right], \\
P_{1}=-\frac{B g \beta \delta^{2}}{2 h}(Z-1)^{2} .
\end{gathered}
$$

Here, $\delta=\frac{h}{l}$ is the dimensionless anisotropy parameter, $Z=\frac{z}{h}$ is a dimensionless variable, normalized with respect to the fluid layer thickness. 


\section{VELOCITY FIELD ANALYSIS}

The analysis of the obtained solution (9) for the velocity components $U, u$, and $w$ has shown that, taking into account the no-slip conditions at the lower boundary of the considered fluid layer, the function $U$ has no zero points on the interval $Z \in(0 ; 1)$, and each function $u$ and $w$ can have no more than one zero point on the same interval. Such values of the fluid layer thickness $Z$ that a velocity component vanishes are here referred to as stagnation points of this component. The profiles of the velocity vector components $u$ and $w$ are shown in Fig. 1 .

Figure 2 shows streamlines in the case of the existence of stagnation points for the velocity vector components $u$ and $w$. Fluid injection is observed at the upper permeable boundary of the layer.

For the layer thickness value corresponding to the stagnation point of the function $u$, the streamline functions change direction, and for the layer thickness value corresponding to the stagnation point of the function $w$, the streamline functions become horizontal.

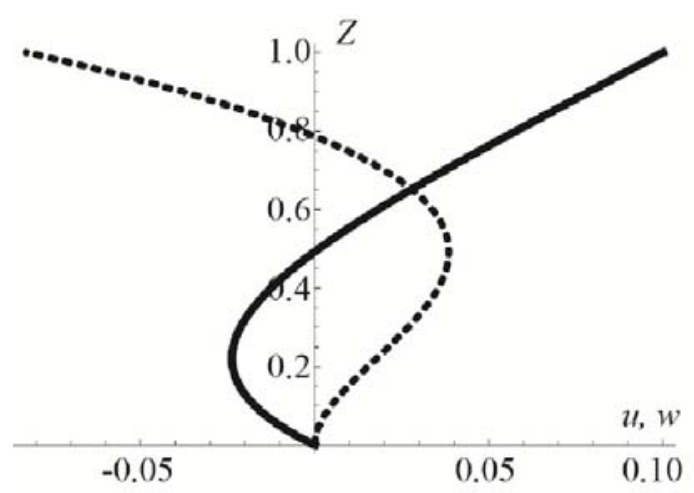

FIGURE 1. The profiles of the velocity components $u$ (solid line) and $w$ (dashed line) for the following parameter values: $g=9.8 \mathrm{~m} / \mathrm{s}^{2}, \beta=1.82 \cdot 10^{-4} 1 / \mathrm{K}, v=10^{-6} \mathrm{~m}^{2} / \mathrm{s}$, $h=5 \mathrm{~m}, \delta=10^{-2}, B=-3 \mathrm{~K} / \mathrm{m}, \Omega=10^{-1} 1 / \mathrm{s}$

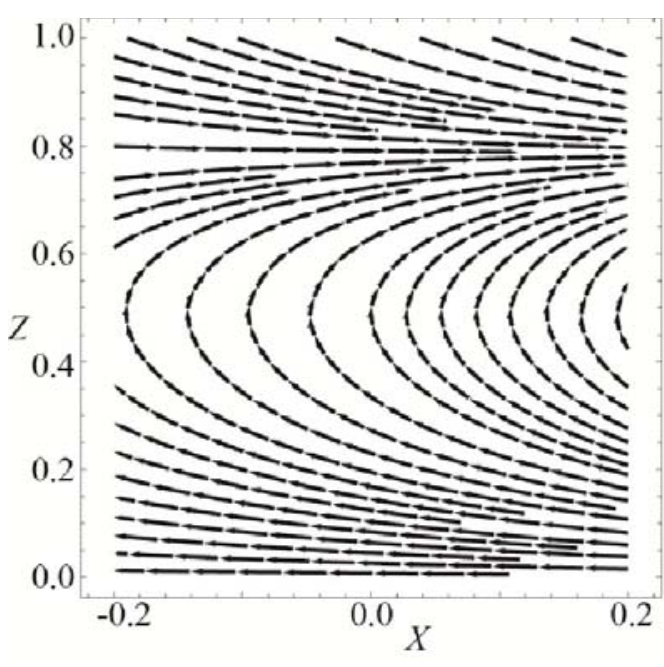

FIGURE 2. The streamlines for the following parameter values: $g=9.8 \mathrm{~m} / \mathrm{s}^{2}, \beta=1.82 \cdot 10^{-4} 1 / \mathrm{K}, v=10^{-6} \mathrm{~m}^{2} / \mathrm{s}$, $h=5 \mathrm{~m}, \delta=10^{-2}, B=-3 \mathrm{~K} / \mathrm{m}, \Omega=10^{-1} 1 / \mathrm{s}$

\section{TEMPERATURE AND PRESSURE FIELD ANALYSIS}

The function $T_{1}$ of the obtained solution (9) is a linear function of a constant sign. If the parameters values $A$ and $B$ determining the heating of the lower boundary are comparable in the order of smallness, the polynomial determining the background temperature function $T_{0}$ has no zero values in the interval $Z \in(0 ; 1)$. Besides, this polynomial $T_{0}$ is a monotonic function of a constant sign, which varies from the value of $A$ at the lower boundary to zero at the upper boundary of the fluid layer. If the parameter $B$ is several orders of magnitude greater than the parameter $A$, the background temperature profile has the form shown in Fig. 3. 


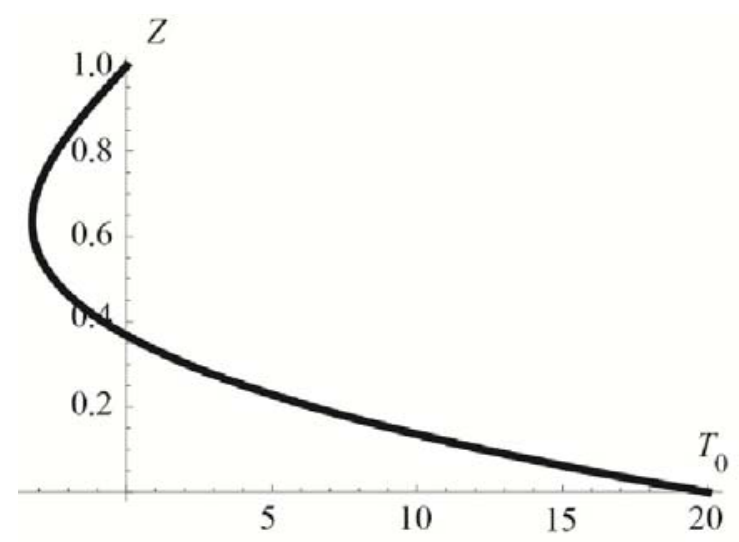

FIGURE 3. The profile of the function $T_{0}$ at the following values of the parameters: $A=2 \cdot 10 \mathrm{~K}, B=-2 \cdot 10^{4} \mathrm{~K} / \mathrm{m}, \delta=10^{-1}$

The background pressure function $P_{0}$ of the partial exact solution (9) for normal values of atmospheric pressure and temperature over the entire thickness of the fluid layer assumes a value approximately equal to that specified at the upper boundary. This function begins to be significantly affected by the thermogravitational flow (the terms with the coefficients $A, B$, and $\Omega$ ) as the boundary condition parameters increase by 4-6 orders of magnitude from the normal conditions assumed, for example, in oceanology.

\section{CONCLUSION}

The paper has presented a new exact solution describing the plane convective Couette flow of a viscous incompressible fluid. Only the lower boundary of the fluid layer is heated according to the quadratic (parabolic) dependence. It has been demonstrated that the occurrence of counterflow regions is caused precisely by a nonzero temperature change along the longitudinal coordinate at the lower boundary of a viscous incompressible fluid layer. The analysis of the solutions is applicable to studies of fluid flows in thin layers.

\section{REFERENCES}

1. G. Z. Gershuni and E. M. Zhukhovitskii, Convective Stability of Incompressible Fluids (Israel Program for Scientific Translations, Keter Publishing House, Jerusalem, 1976).

2. $\quad$ L. D. Landau and E. M. Lifshitz, Fluid Mechanics (Pergamon Press, Oxford, 1987).

3. S. N. Aristov, V. V. Privalova, and E. Yu. Prosviryakov, Vestnik KGTU im. A. N. Tupoleva 71(2), 69-75 (2015).

4. S. N. Aristov, V. V. Privalova, and E. Yu. Prosviryakov, Rus. J. Nonlin. Dyn. 12(2), 167-178 (2016).

5. V. V. Privalova and E. Yu. Prosviryakov, Vestnik KGTU im. A. N. Tupoleva 71(5), 148-153 (2015).

6. V. V. Privalova and E. Yu. Prosviryakov, Rus. J. Nonlin. Dyn. 14(1), 69-79 (2018).

7. C. C. Lin, Arch. Rational Mech. Anal. 1, 391-395 (1958).

8. P. F. Neményi, Advances in Appl. Mech. 11, 123-151 (1951).

9. S. N. Aristov, D. V. Knyazev, and A. D. Polyanin, Theor. Found. Chem. Eng. 43(5), 642-662 (2009). 